\title{
When Equivalence and Bisimulation Join Forces in Probabilistic Automata ${ }^{\star}$
}

\author{
Yuan Feng ${ }^{1,2}$ and Lijun Zhang ${ }^{3}$ \\ 1 University of Technology Sydney, Australia \\ 2 Department of Computer Science and Technology, Tsinghua University, China \\ 3 State Key Laboratory of Computer Science, Institute of Software, Chinese Academy of \\ Sciences
}

\begin{abstract}
Probabilistic automata were introduced by Rabin in 1963 as language acceptors. Two automata are equivalent if and only if they accept each word with the same probability. On the other side, in the process algebra community, probabilistic automata were re-proposed by Segala in 1995 which are more general than Rabin's automata. Bisimulations have been proposed for Segala's automata to characterize the equivalence between them. So far the two notions of equivalences and their characteristics have been studied most independently. In this paper, we consider Segala's automata, and propose a novel notion of distributionbased bisimulation by joining the existing equivalence and bisimilarities. Our bisimulation bridges the two closely related concepts in the community, and provides a uniform way of studying their characteristics. We demonstrate the utility of our definition by studying distribution-based bisimulation metrics, which gives rise to a robust notion of equivalence for Rabin's automata.
\end{abstract}

\section{Introduction}

In 1963, Rabin [28] introduced the model probabilistic automata as language acceptors. In a probabilistic automaton, each input symbol determines a stochastic transition matrix over the state space. Starting with the initial distribution, each word (a sequence of symbols) has a corresponding probability of reaching one of the final states, which is referred to the accepting probability. Two automata are equivalent if and only if they accept each word with the same probability. The corresponding decision algorithm has been extensively studied, see [28, 30, 24, 25].

Markov decision processes (MDPs) were known as early as the 1950s [3], and are a popular modeling formalism used for instance in operations research, automated planning, and decision support systems. In MDPs, each state has a set of enabled actions and each enabled action leads to a distribution over successor states. MDPs have been widely used in the formal verification of randomized concurrent systems, and are now supported by probabilistic model checking tools such as PRISM [26], MRMC [23] and IscasMC [19].

\footnotetext{
* Supported by the National Natural Science Foundation of China (NSFC) under grant No. 61361136002, and Australian Research Council (ARC) under grant Nos. DP130102764 and FT100100218. Y. F. is also supported by the Overseas Team Program of Academy of Mathematics and Systems Science, Chinese Academy of Sciences.
} 
On the other side, in the context of concurrent systems, probabilistic automata were re-proposed by Segala in 1995 [29], which extend MDPs with internal nondeterministic choices. Segala's automata are more general than Rabin's automata, in the sense that each input symbol corresponds to one, or more than one, stochastic transition matrices. Various behavioral equivalences are defined, including strong bisimulations, strong probabilistic bisimulations, and weak bisimulation extensions [29]. These behavioral equivalences are used as powerful tools for state space reduction and hierarchical verification of complex systems. Thus, their decision algorithms $[4,2,22]$ and logical characterizations $[27,12,21]$ are widely studied in the literature.

Equivalences are defined for the specific initial distributions over Rabin's automata, whereas bisimulations are usually defined over states. For Segala's automata, statebased bisimulations have arguably too strong distinguishing power, thus in the recent literature, various relaxations have been proposed. The earliest such formulation is a distribution-based bisimulation in [14], which is defined for Rabin's automata. This is essentially an equivalent characterization of the equivalence in the coinductive manner, as for bisimulations. Recently, in [15], a distribution-based weak bisimulation has been proposed, and the induced distribution-based strong bisimulation is further studied in [20]. It is shown that the distribution-based strong bisimulation agrees with the state-based bisimulations when lifted to distributions.

To the best of the authors' knowledge, even the two notions are closely related, so far their characteristics have been studied independently. As the main contribution of this paper, we consider Segala's probabilistic automata, and propose a novel notion of distribution-based bisimulation by joining the existing equivalence and bisimilarities. We show that for Rabin's probabilistic automata it coincides with equivalences, and for Segala's probabilistic automata, it is reasonably weaker than the existing bisimulation relation. Thus, our bisimulations bridge the two closely related concepts in the community, and provide a uniform way of studying their characteristics.

We demonstrate the utility of our approach by studying distribution-based bisimulation metrics. Bisimulations for probabilistic systems are known to be very sensitive to the transition probabilities: even a tiny perturbation of the transition probabilities will destroy bisimilarity. Thus, bisimulation metrics have been proposed [18]: the distance between any two states are measured, and the smaller the distance is, the more similar they are. If the distance is zero, one then has the classical bisimulation. Because of the nice property of robustness, bisimulation metrics have attracted a lot attentions on MDPs and their extension with continuous state space, see [10, 8, 11, 13, 16, 6, 17, 1,7].

All of the existing bisimulation metrics mentioned above are state-based. On the other side, as states lead to distributions in MDPs, the metrics must be lifted to distributions. In the second part of the paper, we propose a distribution-based bisimulation metric; we consider it being more natural as no lifting of distances is needed. We provide a coinductive definition as well as a fixed point characterization, both of which are used in defining the state-based bisimulation metrics in the literature. We provide a logical characterization for this metric as well, and discuss the relation of our definition and the state-based ones.

A direct byproduct of our bisimulation-based metrics is the notion of equivalence metrics for Rabin's probabilistic automata. As for bisimulation metrics, the equivalence 
metric provides a robust solution for comparing Rabin's automata. To the best of our knowledge, this has not been studied in the literature. We anticipate that more solution techniques developed in one area can inspire solutions for the corresponding problems in the other.

Organization of the paper. We introduce some notations in Section 2. Section 3 recalls the definitions of probabilistic automata, equivalence, and bisimulation relations. We present our distribution-based bisimulation in Section 4, and bisimulation metrics and their logical characterizations in 5. Section 6 concludes the paper.

\section{Preliminaries}

Distributions. For a finite set $S$, a distribution is a function $\mu: S \rightarrow[0,1]$ satisfying $|\mu|:=\sum_{s \in S} \mu(s)=1$. We denote by $\operatorname{Dist}(S)$ the set of distributions over $S$. We shall use $s, r, t, \ldots$ and $\mu, \nu \ldots$ to range over $S$ and $\operatorname{Dist}(S)$, respectively. Given a set of distributions $\left\{\mu_{i}\right\}_{1 \leq i \leq n}$, and a set of positive weights $\left\{p_{i}\right\}_{1 \leq i \leq n}$ such that $\sum_{1 \leq i \leq n} p_{i}=1$, the convex combination $\mu=\sum_{1 \leq i \leq n} p_{i} \cdot \mu_{i}$ is the distribution such that $\mu(s)=\sum_{1 \leq i \leq n} p_{i} \cdot \mu_{i}(s)$ for each $s \in \bar{S}$. The support of $\mu$ is defined by $\operatorname{supp}(\mu):=\{s \in S \mid \mu(s)>0\}$. For an equivalence relation $R$ defined on $S$, we write $\mu R \nu$ if it holds that $\mu(C)=\nu(C)$ for all equivalence classes $C \in S / R$. A distribution $\mu$ is called Dirac if $|\operatorname{supp}(\mu)|=1$, and we let $\delta_{s}$ denote the Dirac distribution with $\delta_{s}(s)=1$.

Note that when $S$ is finite, the distributions $\operatorname{Dist}(S)$ over $S$, when regarded as a subset of $\mathbb{R}^{|S|}$, is both convex and compact. In this paper, when we talk about convergence of distributions, or continuity of relations such as transitions, bisimulations, and pseudometrics between distributions, we are referring to the normal topology of $\mathbb{R}^{|S|}$. For a set $F \subseteq S$, we define the (column) characteristic vector $\eta_{F}$ by letting $\eta_{F}(s)=1$ if $s \in F$, and 0 otherwise.

Pseudometric. A pseudometric over $\operatorname{Dist}(S)$ is a function $d: \operatorname{Dist}(S) \times \operatorname{Dist}(S) \rightarrow$ $[0,1]$ such that (i) $d(\mu, \mu)=0$; (ii) $d(\mu, \nu)=d(\nu, \mu)$; (iii) $d(\mu, \nu)+d(\nu, \omega) \geq d(\mu, \omega)$. In this paper, we assume that a pseudometric is continuous.

\section{Probabilistic Automata and Bisimulations}

\subsection{Probabilistic Automata}

Let $A P$ be a finite set of atomic propositions. We recall the notion of probabilistic automata introduced by Segala [29].

Definition 1 (Probabilistic Automata). A probabilistic automaton is a tuple $\mathcal{A}=$ $(S$, Act $, \rightarrow, L, \alpha)$ where $S$ is a finite set of states, Act is a finite set of actions, $\rightarrow \subseteq$ $S \times \operatorname{Act} \times \operatorname{Dist}(S)$ is a transition relation, $L: S \rightarrow 2^{A P}$ is a labeling function, and $\alpha \in \operatorname{Dist}(S)$ is an initial distribution.

As usual we only consider image-finite probabilistic automata, i.e. for all $s \in S$, the set $\{\mu \mid(s, a, \mu) \in \rightarrow\}$ is finite. A transition $(s, a, \mu) \in \rightarrow$ is denoted by $s \stackrel{a}{\rightarrow} \mu$. We denote by $A c t(s):=\{a \mid s \stackrel{a}{\rightarrow} \mu\}$ the set of enabled actions in $s$. We say $\mathcal{A}$ is input enabled, if $A c t(s)=A c t$ for all $s \in S$. We say $\mathcal{A}$ is an MDP if Act is a singleton. 
Interestingly, a subclass of probabilistic automata were already introduced by Rabin in 1963 [28]; Rabin's probabilistic automata were referred to as reactive automata in [29]. We adopt this convention in this paper.

Definition 2 (Reactive Automata). We say $\mathcal{A}$ is reactive if it is input enabled, and for all $s, L(s) \in\{\emptyset, A P\}$, and $s \stackrel{a}{\rightarrow} \mu \wedge \stackrel{a}{\rightarrow} \mu^{\prime} \Rightarrow \mu=\mu^{\prime}$.

Here the condition $L(s) \in\{\emptyset, A P\}$ implies that the states can be partitioned into two equivalence classes according to their labeling. Below we shall identify $F:=\{s \mid$ $L(s)=A P\}$ as the set of accepting states, a terminology used in reactive automata. In a reactive automaton, each action $a \in A c t$ is enabled precisely once for all $s \in S$, thus inducing a stochastic matrix $M(a)$ satisfying $s \stackrel{a}{\rightarrow} M(a)(s, \cdot)$.

\subsection{Probabilistic Bisimulation and Equivalence}

First, we recall the definition of (strong) probabilistic bisimulation for probabilistic automata [29]. Let $\left\{s \stackrel{a}{\rightarrow} \mu_{i}\right\}_{i \in I}$ be a collection of transitions, and let $\left\{p_{i}\right\}_{i \in I}$ be a collection of probabilities with $\sum_{i \in I} p_{i}=1$. Then $\left(s, a, \sum_{i \in I} p_{i} \cdot \mu_{i}\right)$ is called a combined transition and is denoted by $s \stackrel{a}{\rightarrow} \mathrm{P} \mu$ where $\mu=\sum_{i \in I} p_{i} \cdot \mu_{i}$.

Definition 3 (Probabilistic bisimulation [29]). An equivalence relation $R \subseteq S \times S$ is a probabilistic bisimulation if $s R r$ implies that $L(s)=L(r)$, and for each $s \stackrel{a}{\rightarrow} \mu$, there exists a combined transition $r \stackrel{a}{\rightarrow} \mathrm{p} \nu$ such that $\mu R \nu$.

We write $s \sim_{\mathrm{p}} r$ whenever there is a probabilistic bisimulation $R$ such that $s R r$.

Recently, in [15], a distribution-based weak bisimulation has been proposed, and the induced distribution-based strong bisimulation is further studied in [20]. Their bisimilarity is shown to be the same as $\sim_{\mathrm{P}}$ when lifted to distributions. Below we recall the definition of equivalence for reactive automata introduced by Rabin [28].

Definition 4 (Equivalence for Reactive Automata [28]). Let $\mathcal{A}_{i}=\left(S_{i}, A_{c} t_{i}, \rightarrow_{i}\right.$ $\left., L_{i}, \alpha_{i}\right)$ with $i=1,2$ be two reactive automata with Act $_{1}=$ Act $_{2}=:$ Act, and $F_{i}=\left\{s \in S_{i} \mid L(s)=A P\right\}$ the set of final states for $\mathcal{A}_{i}$. We say $\mathcal{A}_{1}$ and $\mathcal{A}_{2}$ are equivalent if $\mathcal{A}_{1}(w)=\mathcal{A}_{2}(w)$ for each $w \in A c t^{*}$, where $\mathcal{A}_{i}(w):=\alpha_{i} M_{i}\left(a_{1}\right) \ldots M_{i}\left(a_{k}\right) \eta_{F_{i}}$ provided $w=a_{1} \ldots a_{k}$.

Stated in plain english, $\mathcal{A}_{1}$ and $\mathcal{A}_{2}$ with the same set of actions are equivalent iff for an arbitrary input $w$, the probabilities of absorbing in $F_{1}$ and $F_{2}$ are the same.

So far bisimulations and equivalences were studied most independently. The only exception we are aware is [14], in which for Rabin's probabilistic automata, a distributionbased bisimulation is defined that generalizes both equivalence and bisimulations.

Definition 5 (Bisimulation for Reactive Automata [14]). Let $\mathcal{A}_{i}=\left(S_{i}\right.$, Act $_{i}, \rightarrow_{i}$ , $\left.L_{i}, \alpha_{i}\right)$ with $i=1,2$ be two given reactive automata with Act $_{1}=$ Act $_{2}=:$ Act, and $F_{i}$ the set of final states for $\mathcal{A}_{i}$. A relation $R \subseteq \operatorname{Dist}\left(S_{1}\right) \times \operatorname{Dist}\left(S_{2}\right)$ is a bisimulation if for each $\mu R \nu$ it holds (i) $\mu \cdot \eta_{F_{1}}=\nu \cdot \eta_{F_{2}}$, and (ii) $\left(\mu M_{1}(a)\right) R\left(\nu M_{2}(a)\right)$ for all $a \in$ Act.

We write $\mu \sim_{d} \nu$ whenever there is a bisimulation $R$ such that $\mu R \nu$.

It is shown in [14] that two reactive automata are equivalent if and only if their initial distributions are distribution-based bisimilar according to the definition above. 


\section{A Novel Bisimulation Relation}

In this section we introduce a notion of distribution-based bisimulation for Segala's automata by extending the bisimulation defined in [14]. We shall show the compatibility of our definition with previous ones in Subsection 4.1, and some properties of our bisimulation in Subsection 4.2.

For the first step of defining a distribution-based bisimulation, we need to extend the transitions starting from states to those starting from distributions. A natural candidate for such an extension is as follows: for a distribution $\mu$ to perform an action $a$, each state in its support must make a combined $a$-move. However, this definition is problematic, as in Segala's general probabilistic automata, action $a$ may not always be enabled in any support state of $\mu$. In this paper, we deal with this problem by first defining the distribution-based bisimulation (resp. distances) for input enabled automata, for which the transition between distributions can be naturally defined, and then reducing the equivalence (resp. distances) of two distributions in a general probabilistic automata to the bisimilarity (resp. distances) of these distributions in an input enabled automata which is obtained from the original one by adding a dead state.

To make our idea more rigorous, we need some notations. For $A \subseteq A P$ and a distribution $\mu$, we define $\mu(A):=\sum\{\mu(s) \mid L(s)=A\}$, which is the probability of being in those state $s$ with label $A$.

Definition 6. We write $\mu \stackrel{a}{\rightarrow} \mu^{\prime}$ if for each $s \in \operatorname{supp}(\mu)$ there exists $s \stackrel{a}{\rightarrow} \mathrm{P} \mu_{s}$ such that $\mu^{\prime}=\sum_{s} \mu(s) \cdot \mu_{s}$.

We first present our distribution-based bisimulation for input enabled probabilistic automata.

Definition 7. Let $\mathcal{A}=(S$, Act $, \rightarrow, L, \alpha)$ be an input enabled probabilistic automaton. A symmetric relation $R \subseteq \operatorname{Dist}(S) \times \operatorname{Dist}(S)$ is a (distribution-based) bisimulation if $\mu R \nu$ implies that

1. $\mu(A)=\nu(A)$ for each $A \subseteq A P$, and

2. for each $a \in A$ ct, whenever $\mu \stackrel{a}{\rightarrow} \mu^{\prime}$ then there exists a transition $\nu \stackrel{a}{\rightarrow} \nu^{\prime}$ such that $\mu^{\prime} R \nu^{\prime}$.

We write $\mu \sim^{\mathcal{A}} \nu$ if there is a bisimulation $R$ such that $\mu R \nu$.

Obviously, the bisimilarity $\sim^{\mathcal{A}}$ is the largest bisimulation relation.

For probabilistic automata which are not input enabled, we define distribution-based bisimulation with the help of input enabled extension specified as follows.

Definition 8. Let $\mathcal{A}=(S$, Act $, \rightarrow, L, \alpha)$ be a probabilistic automaton over AP. The input enabled extension of $\mathcal{A}$, denoted by $\mathcal{A}_{\perp}$, is defined as an (input enabled) probabilistic automaton $\left(S_{\perp}\right.$, Act $\left., \rightarrow^{\perp}, L_{\perp}, \alpha\right)$ over $A P_{\perp}$ where

1. $S_{\perp}=S \cup\{\perp\}$ where $\perp$ is a dead state not in $S$;

2. $A P_{\perp}=A P \cup\{$ dead $\}$ with dead $\notin A P$;

3. $\rightarrow^{\perp}=\rightarrow \cup\left\{\left(s, a, \delta_{\perp}\right) \mid a \notin A c t(s)\right\} \cup\left\{\left(\perp, a, \delta_{\perp}\right) \mid a \in A c t\right\}$;

4. $L_{\perp}(s)=L(s)$ for any $s \in S$, and $L_{\perp}(\perp)=\{$ dead $\}$. 
Definition 9. Let $\mathcal{A}$ be a probabilistic automaton which is not input enabled. Then $\mu$ and $\nu$ are bisimilar, denoted by $\mu \sim^{\mathcal{A}} \nu$, if $\mu \sim^{\mathcal{A}_{\perp}} \nu$ in $\mathcal{A}_{\perp}$.

We always omit the superscript $\mathcal{A}$ in $\sim^{\mathcal{A}}$ when no confusion arises.

\subsection{Compatibility}

In this section we instantiate appropriate labeling functions and show that our notion of bisimilarity is a conservative extension of both probabilistic bisimulation [28] and equivalence relations [14].

Lemma 1. Let $\mathcal{A}$ be a probabilistic automaton where $A P=A c t$, and $L(s)=\operatorname{Act}(s)$ for each s. Then, $\mu \sim_{\mathrm{p}} \nu$ implies $\mu \sim \nu$.

Proof. First, it is easy to see that for a given probabilistic automata $\mathcal{A}$ with $A P=A c t$ and $L(s)=\operatorname{Act}(s)$ for each $s$, and distributions $\mu$ and $\nu$ in $\operatorname{Dist}(S), \mu \sim_{\mathrm{p}} \nu$ in $\mathcal{A}$ if and only if $\mu \sim_{\mathrm{p}} \nu$ in the input enabled extension $\mathcal{A}_{\perp}$. Thus we can assume without loss of any generality that $\mathcal{A}$ itself is input enabled.

It suffices to show that the symmetric relation

$$
R=\left\{(\mu, \nu) \mid \mu \sim_{\mathrm{P}} \nu\right\}
$$

is a bisimulation. For each $A \subseteq A c t$, let $S(A)=\{s \in S \mid L(s)=A\}$. Then $S(A)$ is the disjoint union of some equivalence classes of $\sim_{\mathrm{p}}$; that is, $S(A)=\cup\left\{M \in S / \sim_{\mathrm{p}} \mid\right.$ $M \cap S(A) \neq \emptyset\}$. Suppose $\mu \sim_{\mathrm{P}} \nu$. Then for any $M \in S / \sim_{\mathrm{P}}, \mu(M)=\nu(M)$, hence $\mu(A)=\mu(S(A))=\nu(S(A))=\nu(A)$.

Let $\mu \stackrel{a}{\rightarrow} \mu^{\prime}$. Then for any $s \in S$ there exists $s \stackrel{a}{\rightarrow} \mathrm{P} \mu_{s}$ such that

$$
\mu^{\prime}=\sum_{s \in S} \mu(s) \cdot \mu_{s}
$$

Now for each $t \in S$, let $[t]_{\sim_{\mathrm{P}}}$ be the equivalence class of $\sim_{\mathrm{P}}$ which contains $t$. Then for every $s \in[t]_{\sim_{\mathrm{P}}}$, to match the transition $s \stackrel{a}{\rightarrow} \mathrm{P} \mu_{s}$ there exists some $\nu_{t}^{s}$ such that $t \stackrel{a}{\rightarrow} \mathrm{p} \nu_{t}^{s}$ and $\mu_{s} \sim \sim_{\mathrm{p}} \nu_{t}^{s}$. Let

$$
\nu_{t}=\sum_{s \in[t]_{\sim P}} \frac{\mu(s)}{\mu\left([t]_{\sim_{P}}\right)} \cdot \nu_{t}^{s} .
$$

Then we have $t \stackrel{a}{\rightarrow} \mathrm{p} \nu_{t}$, and $\nu \stackrel{a}{\rightarrow} \nu^{\prime}$ where

$$
\nu^{\prime}:=\sum_{t \in S} \nu(t) \cdot \nu_{t}
$$

It remains to prove $\mu^{\prime} \sim_{\mathrm{P}} \nu^{\prime}$. For any $M \in S / \sim_{\mathrm{P}}$, since $\mu_{s} \sim_{\mathrm{P}} \nu_{t}^{s}$ we have

$$
\nu_{t}(M)=\sum_{s \in[t]_{\sim_{\mathrm{P}}}} \frac{\mu(s)}{\mu\left([t]_{\sim_{\mathrm{P}}}\right)} \nu_{t}^{s}(M)=\sum_{s \in[t]_{\sim_{\mathrm{P}}}} \frac{\mu(s)}{\mu\left([t]_{\sim_{\mathrm{P}}}\right)} \mu_{s}(M) .
$$


Thus

$$
\begin{aligned}
\nu^{\prime}(M) & =\sum_{t \in S} \nu(t) \sum_{s \in[t]_{\sim_{\mathrm{P}}}} \frac{\mu(s)}{\mu\left([t]_{\sim_{\mathrm{P}}}\right)} \mu_{s}(M) \\
& =\sum_{s \in S} \mu(s) \mu_{s}(M) \sum_{t \in[s]_{\sim_{\mathrm{P}}}} \frac{\nu(t)}{\nu\left([s]_{\sim_{\mathrm{P}}}\right)} \\
& =\sum_{s \in S} \mu(s) \mu_{s}(M)=\mu^{\prime}(M)
\end{aligned}
$$

where for the second equality we have used the fact that $\mu\left([t]_{\sim_{P}}\right)=\nu\left([s]_{\sim_{P}}\right)$ for any $s \sim$ P

Probabilistic bisimulation is defined over states inside one automaton, whereas equivalence and distribution for reactive automata are defined over two automata. However, they can be connected by the notion of direct sum of two automata, which is the automaton obtained by considering the disjoint union of states, edges and labeling functions respectively.

Lemma 2. Let $\mathcal{A}_{1}$ and $\mathcal{A}_{2}$ be two reactive automata with the same set of actions Act. Let $F_{i}=\left\{s \in S_{i} \mid L(s)=A P\right\}$. Then, the following are equivalent:

1. $\mathcal{A}_{1}$ and $\mathcal{A}_{2}$ are equivalent,

2. $\alpha_{1} \sim_{d} \alpha_{2}$,

3. $\alpha_{1} \sim \alpha_{2}$ in their direct sum.

Proof. The equivalence between (1) and (2) is shown in [14]. The equivalence between (2) and (3) is straightforward, as for reactive automata our definition degenerates to Definition 5.

To conclude this section, we present an example to show that our bisimilarity is strictly weaker than $\sim_{\mathrm{P}}$.

Example 1. Consider the example probabilistic automaton depicted in Fig. 1, which is inspired from an example in [14]. Let $A P=\operatorname{Act}=\{a\}, L(s)=\operatorname{Act}(s)$ for each $s$, and $\varepsilon_{1}=\varepsilon_{2}=0$. We argue that $q \chi_{\mathrm{P}} q^{\prime}$. Otherwise, note $q \stackrel{a}{\rightarrow} \frac{1}{2} \delta_{r_{1}}+\frac{1}{2} \delta_{r_{2}}$ and $q^{\prime} \stackrel{a}{\rightarrow} \delta_{r^{\prime}}$. Then we must have $r^{\prime} \sim_{\mathrm{P}} r_{1} \sim_{\mathrm{P}} r_{2}$. This is impossible, as $r_{1} \stackrel{a}{\rightarrow} \frac{2}{3} \delta_{s_{1}}+\frac{1}{3} \delta_{s_{2}}$ and $r^{\prime} \stackrel{a}{\rightarrow} \frac{1}{2} \delta_{s_{1}^{\prime}}+\frac{1}{2} \delta_{s_{2}^{\prime}}$, but $s_{1} \sim_{\mathrm{P}} s_{1}^{\prime} \chi_{\mathrm{P}} s_{2} \sim_{\mathrm{P}} s_{2}^{\prime}$.

However, by our definition of bisimulation, the Dirac distributions $\delta_{q}$ and $\delta_{q^{\prime}}$ are indeed bisimilar. The reason is, we have the following transition

$$
\frac{1}{2} \delta_{r_{1}}+\frac{1}{2} \delta_{r_{2}} \stackrel{a}{\rightarrow} \frac{1}{3} \delta_{s_{1}}+\frac{1}{6} \delta_{s_{2}}+\frac{1}{6} \delta_{s_{3}}+\frac{1}{3} \delta_{s_{4}},
$$

and it is easy to check $\delta_{s_{1}} \sim \delta_{s_{3}} \sim \delta_{s_{1}^{\prime}}$ and $\delta_{s_{2}} \sim \delta_{s_{4}} \sim \delta_{s_{2}^{\prime}}$. Thus we have $\frac{1}{2} \delta_{r_{1}}+$ $\frac{1}{2} \delta_{r_{2}} \sim \delta_{r^{\prime}}$, and finally $\delta_{q} \sim \delta_{q^{\prime}}$. 


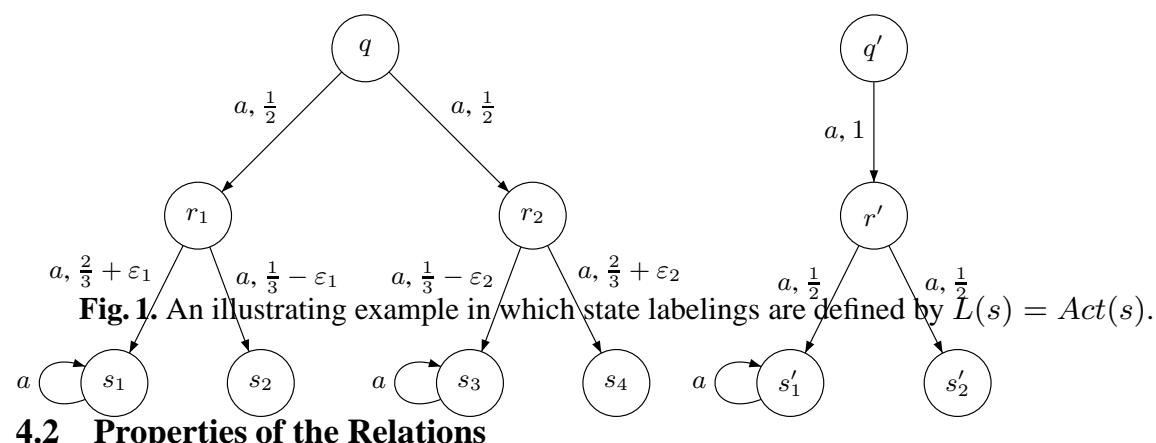

4.2 Properties of the Relations

In the following, we show that the notion of bisimilarity is in harmony with the linear combination and the limit of distributions.

Definition 10. A binary relation $R \subseteq \operatorname{Dist}(S) \times \operatorname{Dist}(S)$ is said to be

- linear, if for any finite set I and any probabilistic distribution $\left\{p_{i}\right\}_{i \in I}, \mu_{i} R \nu_{i}$ for each $i$ implies $\left(\sum_{i \in I} p_{i} \cdot \mu_{i}\right) R\left(\sum_{i \in I} p_{i} \cdot \nu_{i}\right)$;

- continuous, iffor any convergent sequences of distributions $\left\{\mu_{i}\right\}_{i}$ and $\left\{\nu_{i}\right\}_{i}, \mu_{i} R \nu_{i}$ for each $i$ implies $\left(\lim _{i} \mu_{i}\right) R\left(\lim _{i} \nu_{i}\right)$;

- left-decomposable, if $\left(\sum_{i \in I} p_{i} \cdot \mu_{i}\right) R \nu$, where $0<p_{i} \leq 1$ and $\sum_{i \in I} p_{i}=1$, then $\nu$ can be written as $\sum_{i \in I} p_{i} \cdot \nu_{i}$ such that $\mu_{i} R \nu_{i}$ for every $i \in I$.

- left-convergent, if $\left(\lim _{i} \mu_{i}\right) R \nu$, then for any $i$ we have $\mu_{i} R \nu_{i}$ for some $\nu_{i}$ with $\lim _{i} \nu_{i}=\nu$.

We prove below that our transition relation between distributions satisfies these properties.

Lemma 3. For an input enabled probabilistic automata, the transition relation $\stackrel{a}{\rightarrow}$ between distributions is linear, continuous, left-decomposable, and left-convergent.

Proof. - Linearity. Let $I$ be a finite index set and $\left\{p_{i} \mid i \in I\right\}$ a probabilistic distribution on $I$. Suppose $\mu_{i} \stackrel{a}{\rightarrow} \nu_{i}$ for each $i \in I$. Then by definition, for each $s$ there exists $s \stackrel{a}{\rightarrow} \mathrm{P} \mu_{s}^{i}$ such that $\nu_{i}=\sum_{s} \mu_{i}(s) \cdot \mu_{s}^{i}$. Now let $\mu=\sum_{i \in I} p_{i} \cdot \mu_{i}$. Then for each $s \in \operatorname{supp}(\mu)$,

$$
s \stackrel{a}{\rightarrow} \mathrm{P} \mu_{s}:=\sum_{i \in I} \frac{p_{i} \mu_{i}(s)}{\mu(s)} \cdot \mu_{s}^{i} .
$$

On the other hand, we check that

$$
\nu:=\sum_{i \in I} p_{i} \cdot \nu_{i}=\sum_{s \in S} \sum_{i \in I} p_{i} \mu_{i}(s) \cdot \mu_{s}^{i}=\sum_{s \in S} \mu(s) \cdot \mu_{s} .
$$

Thus $\mu \stackrel{a}{\rightarrow} \nu$ as expected.

- Continuity. Suppose $\mu_{i} \stackrel{a}{\rightarrow} \nu_{i}$ for each $i \in I$, and $\lim _{i} \mu_{i}=\mu$. By definition, for each $s$ there exists $s \stackrel{a}{\rightarrow} \mathrm{p} \mu_{s}^{i}$ such that $\nu_{i}=\sum_{s} \mu_{i}(s) \cdot \mu_{s}^{i}$. Note that $\operatorname{Dist}(S)$ is a 
compact set. For each $s$ we can choose a convergent subsequence $\left\{\mu_{s}^{i_{k}}\right\}_{k}$ of $\left\{\mu_{s}^{i}\right\}_{i}$ such that $\lim _{k} \mu_{s}^{i_{k}}=\mu_{s}$ for some $\mu_{s}$. Then $s \stackrel{a}{\rightarrow} \mu_{s}$, and

$$
\mu \stackrel{a}{\rightarrow} \nu:=\sum_{s \in S} \mu(s) \cdot \mu_{s}
$$

Note that for each $k$,

$$
\left\|\nu_{i_{k}}-\nu\right\|_{1} \leq\left\|\mu_{i_{k}}-\mu\right\|_{1}+\sum_{s \in S} \mu(s)\left\|\mu_{s}^{i_{k}}-\mu_{s}\right\|_{1}
$$

where $\|\cdot\|_{1}$ denotes the $l_{1}$-norm. We have $\nu=\lim _{k} \nu_{i_{k}}$ by the assumption that $\lim _{i} \mu_{i}=\mu$. Thus $\lim _{i} \nu_{i}=\nu$, as $\left\{\nu_{i}\right\}_{i}$ itself converges.

- Left-decomposability. Let $\mu:=\left(\sum_{i \in I} p_{i} \cdot \mu_{i}\right) \stackrel{a}{\rightarrow} \nu$. Then by definition, for each $s$ there exists $s \stackrel{a}{\rightarrow} \mathrm{P} \mu_{s}$ such that $\nu=\sum_{s} \mu(s) \cdot \mu_{s}$. Thus

$$
\mu_{i} \stackrel{a}{\rightarrow} \nu_{i}:=\sum_{s \in S} \mu_{i}(s) \cdot \mu_{s}
$$

Finally, it is easy to show that $\sum_{i \in I} p_{i} \cdot \nu_{i}=\nu$.

- Left-convergence. Similar to the last case.

Theorem 1. The bisimilarity relation $\sim$ is both linear and continuous.

Proof. Note that if $\mu_{i} \in \operatorname{Dist}(S)$ for any $i$, then both $\sum_{i} p_{i} \cdot \mu_{i}$ and $\lim _{i} \mu_{i}$ (if exists) are again in $\operatorname{Dist}(S)$. Thus we need only consider the case when the automata is input enabled.

- Linearity. It suffices to show that the symmetric relation

$$
R=\left\{\left(\sum_{i \in I} p_{i} \cdot \mu_{i}, \sum_{i \in I} p_{i} \cdot \nu_{i}\right) \mid I \text { finite }, \sum_{i \in I} p_{i}=1, \forall i .\left(p_{i} \geq 0 \wedge \mu_{i} \sim \nu_{i}\right)\right\}
$$

is a bisimulation. Let $\mu=\sum_{i \in I} p_{i} \cdot \mu_{i}, \nu=\sum_{i \in I} p_{i} \cdot \nu_{i}$, and $\mu R \nu$. Then for any $A \subseteq A P$,

$$
\mu(A)=\sum_{i \in I} p_{i} \cdot \mu_{i}(A)=\sum_{i \in I} p_{i} \cdot \nu_{i}(A)=\nu(A) .
$$

Now suppose $\mu \stackrel{a}{\rightarrow} \mu^{\prime}$. Then by Lemma 3 (left-decomposability), for each $i \in I$ we have $\mu_{i} \stackrel{a}{\rightarrow} \mu_{i}^{\prime}$ for some $\mu_{i}^{\prime}$ such that $\mu^{\prime}=\sum_{i} p_{i} \cdot \mu_{i}^{\prime}$. From the assumption that $\mu_{i} \sim \nu_{i}$, we derive $\nu_{i} \stackrel{a}{\rightarrow} \nu_{i}^{\prime}$ with $\mu_{i}^{\prime} \sim \nu_{i}^{\prime}$ for each $i$. Thus $\nu \stackrel{a}{\rightarrow} \nu^{\prime}:=\sum_{i} p_{i} \cdot \nu_{i}^{\prime}$ by Lemma 3 again (linearity). Finally, it is obvious that $\left(\mu^{\prime}, \nu^{\prime}\right) \in R$.

- Continuity. It suffices to show that the symmetric relation

$$
R=\left\{(\mu, \nu) \mid \forall i \geq 1, \mu_{i} \sim \nu_{i}, \lim _{i} \mu_{i}=\mu, \text { and } \lim _{i} \nu_{i}=\nu\right\}
$$

is a bisimulation. First, for any $A \subseteq A P$, we have

$$
\mu(A)=\lim _{i} \mu_{i}(A)=\lim _{i} \nu_{i}(A)=\nu(A) .
$$


Let $\mu \stackrel{a}{\rightarrow} \mu^{\prime}$. By Lemma 3 (left-convergence), for any $i$ we have $\mu_{i} \stackrel{a}{\rightarrow} \mu_{i}^{\prime}$ with $\lim _{i} \mu_{i}^{\prime}=\mu^{\prime}$. To match the transitions, we have $\nu_{i} \stackrel{a}{\rightarrow} \nu_{i}^{\prime}$ such that $\mu_{i}^{\prime} \sim \nu_{i}^{\prime}$. Note that $\operatorname{Dist}(S)$ is a compact set. We can choose a convergent subsequence $\left\{\nu_{i_{k}}^{\prime}\right\}_{k}$ of $\left\{\nu_{i}^{\prime}\right\}_{i}$ such that $\lim _{k} \nu_{i_{k}}^{\prime}=\nu^{\prime}$ for some $\nu^{\prime}$. From and fact that $\lim _{i} \nu_{i}=\nu$ and Lemma 3 (continuity), it holds $\nu \stackrel{a}{\rightarrow} \nu^{\prime}$ as well. Finally, it is easy to see that $\left(\mu^{\prime}, \nu^{\prime}\right) \in R$.

In general, our definition of bisimilarity is not left-decomposable. This is in sharp contrast with the bisimulations defined by using the lifting technique [9]. However, this should not be regarded as a shortcoming; actually it is the key requirement we abandon in this paper, which makes our definition reasonably weak. This has been clearly illustrated in Example 1.

\section{Bisimulation Metrics}

We present distribution-based bisimulation metrics with discounting factor $\gamma \in(0,1]$ in this section. Three different ways of defining bisimulation metrics between states exist in the literature: one coinductive definition based on bisimulations [34, 32, 33, 13], one based on the maximal logical differences $[10,11,31]$, and one on fixed point $[8$, $31,16]$. We propose all the three versions for our distribution-based bisimulations with discounting. Moreover, we show that they coincide. We fix a discount factor $\gamma \in(0,1]$ throughout this section. For any $\mu, \nu \in \operatorname{Dist}(S)$, we define the distance

$$
d_{A P}(\mu, \nu):=\frac{1}{2} \sum_{A \subseteq A P}|\mu(A)-\nu(A)| .
$$

Then it is easy to check that

$$
d_{A P}(\mu, \nu)=\max _{\mathcal{B} \subseteq 2^{A P}}\left|\sum_{A \in \mathcal{B}} \mu(A)-\sum_{A \in \mathcal{B}} \nu(A)\right|=\max _{\mathcal{B} \subseteq 2^{A P}}\left[\sum_{A \in \mathcal{B}} \mu(A)-\sum_{A \in \mathcal{B}} \nu(A)\right] .
$$

\subsection{A Direct Approach}

Definition 11. Let $\mathcal{A}=(S$, Act $, \rightarrow, L, \alpha)$ be an input enabled probabilistic automaton. A family of symmetric relations $\left\{R_{\varepsilon} \mid \varepsilon \geq 0\right\}$ over Dist $(S)$ is a (discounted) approximate bisimulation if for any $\varepsilon \geq 0$ and $\mu R_{\varepsilon} \nu$, we have

1. $d_{A P}(\mu, \nu) \leq \varepsilon$

2. for each $a \in A$ Act, $\mu \stackrel{a}{\rightarrow} \mu^{\prime}$ implies that there exists a transition $\nu \stackrel{a}{\rightarrow} \nu^{\prime}$ such that $\mu^{\prime} R_{\varepsilon / \gamma} \nu^{\prime}$.

We write $\mu \sim_{\varepsilon}^{\mathcal{A}} \nu$ whenever there is an approximate bisimulation $\left\{R_{\varepsilon} \mid \varepsilon \geq 0\right\}$ such that $\mu R_{\varepsilon} \nu$. For any two distributions $\mu$ and $\nu$, we define the bisimulation distance of $\mu$ and $\nu$ as

$$
D_{b}^{\mathcal{A}}(\mu, \nu)=\inf \left\{\varepsilon \geq 0 \mid \mu \sim_{\varepsilon}^{\mathcal{A}} \nu\right\}
$$


Again, the approximate bisimulation and bisimulation distance of distributions in a general probabilistic automaton can be defined in terms of the corresponding notions in the input enabled extension; that is, $\mu \sim_{\varepsilon}^{\mathcal{A}} \nu$ if $\mu \sim_{\varepsilon}^{\mathcal{A}_{\perp}} \nu$, and $D_{b}^{\mathcal{A}}(\mu, \nu):=D_{b}^{\mathcal{A}_{\perp}}(\mu, \nu)$. We always omit the superscripts for simplicity if no confusion arises.

It is standard to show that the family $\left\{\sim_{\varepsilon} \mid \varepsilon \geq 0\right\}$ is itself an approximate bisimulation. The following lemma collects some more properties of $\sim_{\varepsilon}$.

Lemma 4. 1. For each $\varepsilon$, the $\varepsilon$-bisimilarity $\sim_{\varepsilon}$ is both linear and continuous.

2. If $\mu \sim_{\varepsilon_{1}} \nu$ and $\nu \sim_{\varepsilon_{2}} \omega$, then $\mu \sim_{\varepsilon_{1}+\varepsilon_{2}} \omega$;

3. $\sim_{\varepsilon_{1}} \subseteq \sim_{\varepsilon_{2}}$ whenever $\varepsilon_{1} \leq \varepsilon_{2}$.

Proof. The proof of item 1 is similar to Theorem 1. For item 2, it suffices to show that $\left\{R_{\varepsilon} \mid \varepsilon \geq 0\right\}$ where $R_{\varepsilon}=\bigcup_{\varepsilon_{1}+\varepsilon_{2}=\varepsilon}\left(\sim_{\varepsilon_{1}} \circ \sim_{\varepsilon_{2}}\right)$ is an approximate bisimulation (in the extended automata, if necessary), which is routine. For item 3, suppose $\varepsilon_{2}>0$. Then it is easy to show $\left\{R_{\varepsilon} \mid \varepsilon \geq 0\right\}, R_{\varepsilon}=\sim_{\varepsilon \varepsilon_{1} / \varepsilon_{2}}$, is an approximate bisimulation. Now if $\mu \sim_{\varepsilon_{1}} \nu$, that is, $\mu \sim_{\varepsilon_{2} \varepsilon_{1} / \varepsilon_{2}} \nu$, then $\mu R_{\varepsilon_{2}} \nu$, and thus $\mu \sim_{\varepsilon_{2}} \nu$ as required.

The following theorem states that the infimum in the definition Eq. (1) of bisimulation distance can be replaced by minimum; that is, the infimum is achievable.

Theorem 2. For any $\mu, \nu \in \operatorname{Dist}(S), \mu \sim_{D_{b}(\mu, \nu)} \nu$.

Proof. By definition, we need to prove $\mu \sim_{D_{b}(\mu, \nu)} \nu$ in the extended automaton. We first prove that for any $\varepsilon \geq 0$, the symmetric relations $\left\{R_{\varepsilon} \mid \varepsilon \geq 0\right\}$ where

$$
R_{\varepsilon}=\left\{(\mu, \nu) \mid \mu \sim_{\varepsilon_{i}} \nu \text { for each } \varepsilon_{1} \geq \varepsilon_{2} \geq \cdots \geq 0, \text { and } \lim _{i \rightarrow \infty} \varepsilon_{i}=\varepsilon\right\}
$$

is an approximate bisimulation. Suppose $\mu R_{\varepsilon} \nu$. Since $\mu \sim_{\varepsilon_{i}} \nu$ we have $d_{A P}(\mu, \nu) \leq \varepsilon_{i}$ for each $i$. Thus $d_{A P}(\mu, \nu) \leq \varepsilon$ as well. Furthermore, if $\mu \stackrel{a}{\rightarrow} \mu^{\prime}$, then for any $i \geq 1$, $\nu \stackrel{a}{\rightarrow} \nu_{i}$ and $\mu^{\prime} \sim_{\varepsilon_{i} / \gamma} \nu_{i}$. Since $\operatorname{Dist}(S)$ is compact, there exists a subsequence $\left\{\nu_{i_{k}}\right\}_{k}$ of $\left\{\nu_{i}\right\}_{i}$ such that $\lim _{k} \nu_{i_{k}}=\nu^{\prime}$ for some $\nu^{\prime}$. We claim that

$-\nu \stackrel{a}{\rightarrow} \nu^{\prime}$. This follows from the continuity of the transition $\stackrel{a}{\rightarrow}$, Lemma 3 .

- For each $k \geq 1, \mu^{\prime} \sim_{\varepsilon_{i_{k}} / \gamma} \nu^{\prime}$. Suppose conversely that $\mu^{\prime} \chi_{\varepsilon_{i_{k}} / \gamma} \nu^{\prime}$ for some $k$. Then by the continuity of $\sim_{\varepsilon_{i_{k}} / \gamma}$, we have $\mu^{\prime} \chi_{\varepsilon_{i_{k} / \gamma}} \nu_{j}$ for some $j \geq i_{k}$. This contradicts the fact that $\mu^{\prime} \sim_{\varepsilon_{j} / \gamma} \nu_{j}$ and Lemma 4(3). Thus $\mu^{\prime} R_{\varepsilon / \gamma} \nu^{\prime}$ as required.

Finally, it is direct from definition that there exists a decreasing sequence $\left\{\varepsilon_{i}\right\}_{i}$ such that $\lim _{i} \varepsilon_{i}=D_{b}(\mu, \nu)$ and $\mu \sim_{\varepsilon_{i}} \nu$ for each $i$. Then the theorem follows.

A direct consequence of the above theorem is that the bisimulation distance between two distributions vanishes if and only if they are bisimilar.

Corollary 1. For any $\mu, \nu \in \operatorname{Dist}(S), \mu \sim \nu$ if and only if $D_{b}(\mu, \nu)=0$.

Proof. Direct from Theorem 2, by noting that $\sim=\sim_{0}$.

The next theorem shows that $D_{b}$ is indeed a pseudometric.

Theorem 3. The bisimulation distance $D_{b}$ is a pseudometric on Dist $(S)$. 
Proof. We need only to prove that $D_{b}$ satisfies the triangle inequality

$$
D_{b}(\mu, \nu)+D_{b}(\nu, \omega) \geq D_{b}(\mu, \omega) .
$$

By Theorem 2, we have $\mu \sim_{D_{b}(\mu, \nu)} \nu$ and $\nu \sim_{D_{b}(\nu, \omega)} \omega$. Then the result follows from Lemma 4(2).

\subsection{Modal Characterization of the Bisimulation Metrics}

We now present a Hennessy-Milner type modal logic motivated by $[10,11]$ to characterize the distance between distributions.

Definition 12. The class $\mathcal{L}_{m}$ of modal formulae over AP, ranged over by $\varphi, \varphi_{1}, \varphi_{2}$, etc, is defined by the following grammar:

$$
\varphi::=\mathcal{B}|\varphi \oplus p| \neg \varphi\left|\bigwedge_{i \in I} \varphi_{i}\right|\langle a\rangle \varphi
$$

where $\mathcal{B} \subseteq 2^{A P}, p \in[0,1], a \in A c t$, and $I$ is an index set.

Given an input enabled probabilistic automaton $\mathcal{A}=(S, A c t, \rightarrow, L, \alpha)$ over $A P$, instead of defining the satisfaction relation $\models$ for the qualitative setting, the (discounted) semantics of the logic $\mathcal{L}_{m}$ is given in terms of functions from $\operatorname{Dist}(S)$ to $[0,1]$. For any formula $\varphi \in \mathcal{L}_{m}$, the satisfaction function of $\varphi$, denoted by $\varphi$ again for simplicity, is defined in a structural induction way as follows:

- $\mathcal{B}(\mu):=\sum_{A \in \mathcal{B}} \mu(A)$

- $(\varphi \oplus p)(\mu):=\min \{\varphi(\mu)+p, 1\}$

- $(\neg \varphi)(\mu):=1-\varphi(\mu)$;

- $\left(\bigwedge_{i \in I} \varphi_{i}\right)(\mu):=\inf _{i \in I} \varphi_{i}(\mu)$;

- $(\langle a\rangle \varphi)(\mu):=\sup _{\mu \stackrel{a}{\rightarrow} \mu^{\prime}} \gamma \cdot \varphi\left(\mu^{\prime}\right)$.

Lemma 5. For any $\varphi \in \mathcal{L}_{m}, \varphi: \operatorname{Dist}(S) \rightarrow[0,1]$ is a continuous function.

Proof. We prove by induction on the structure of $\varphi$. The basis case when $\varphi \equiv \mathcal{B}$ is obvious. The case of $\varphi \equiv \varphi^{\prime} \oplus p, \varphi \equiv \neg \varphi^{\prime}$, and $\varphi \equiv \bigwedge_{i \in I} \varphi_{i}$ are all easy from induction. In the following we only consider the case when $\varphi \equiv\langle a\rangle \varphi^{\prime}$.

Take arbitrarily $\left\{\mu_{i}\right\}_{i}$ with $\lim _{i} \mu_{i}=\mu$. We need to show there exists a subsequence $\left\{\mu_{i_{k}}\right\}_{k}$ of $\left\{\mu_{i}\right\}_{i}$ such that $\lim _{k} \varphi\left(\mu_{i_{k}}\right)=\varphi(\mu)$. Take arbitrarily $\varepsilon>0$.

- Let $\mu^{*} \in \operatorname{Dist}(S)$ such that $\mu \stackrel{a}{\rightarrow} \mu^{*}$ and $\varphi(\mu) \leq \gamma \cdot \varphi^{\prime}\left(\mu^{*}\right)+\varepsilon / 2$. We have from the left-convergence of $\stackrel{a}{\rightarrow}$ that $\mu_{i} \stackrel{a}{\rightarrow} \nu_{i}$ for some $\nu_{i}$, and $\lim _{i} \nu_{i}=\mu^{*}$. By induction, $\varphi^{\prime}$ is a continuous function. Thus we can find $N_{1} \geq 1$ such that for any $i \geq N_{1},\left|\varphi^{\prime}\left(\mu^{*}\right)-\varphi^{\prime}\left(\nu_{i}\right)\right|<\varepsilon / 2 \gamma$.

- For each $i \geq 1$, let $\mu_{i}^{*} \in \operatorname{Dist}(S)$ such that $\mu_{i} \stackrel{a}{\rightarrow} \mu_{i}^{*}$ and $\varphi\left(\mu_{i}\right) \leq \gamma \cdot \varphi^{\prime}\left(\mu_{i}^{*}\right)+\varepsilon / 2$. Then we have $\mu \stackrel{a}{\rightarrow} \nu^{*}$ with $\nu^{*}=\lim _{k} \mu_{i_{k}}^{*}$ for some convergent subsequence $\left\{\mu_{i_{k}}^{*}\right\}_{k}$ of $\left\{\mu_{i}^{*}\right\}_{i}$. Again, from the induction that $\varphi^{\prime}$ is continuous, we can find $N_{2} \geq 1$ such that for any $k \geq N_{2},\left|\varphi^{\prime}\left(\mu_{i_{k}}^{*}\right)-\varphi^{\prime}\left(\nu^{*}\right)\right|<\varepsilon / 2 \gamma$. 
Let $N=\max \left\{N_{1}, N_{2}\right\}$. Then for any $k \geq N$, we have from $\mu \stackrel{a}{\rightarrow} \nu^{*}$ that

$$
\begin{aligned}
\varphi\left(\mu_{i_{k}}\right)-\varphi(\mu) & \leq \gamma\left[\varphi^{\prime}\left(\mu_{i_{k}}^{*}\right)-\varphi^{\prime}\left(\nu^{*}\right)\right]+\gamma \cdot \varphi^{\prime}\left(\nu^{*}\right)-\varphi(\mu)+\varepsilon / 2 \\
& \leq \gamma\left[\varphi^{\prime}\left(\mu_{i_{k}}^{*}\right)-\varphi^{\prime}\left(\nu^{*}\right)\right]+\varepsilon / 2<\varepsilon .
\end{aligned}
$$

Similarly, from $\mu_{i_{k}} \stackrel{a}{\rightarrow} \nu_{i_{k}}$ we have

$$
\begin{aligned}
\varphi(\mu)-\varphi\left(\mu_{i_{k}}\right) & \leq \gamma\left[\varphi^{\prime}\left(\mu^{*}\right)-\varphi^{\prime}\left(\nu_{i_{k}}\right)\right]+\gamma \cdot \varphi^{\prime}\left(\nu_{i_{k}}\right)-\varphi\left(\mu_{i_{k}}\right)+\varepsilon / 2 \\
& \leq \gamma\left[\varphi^{\prime}\left(\mu^{*}\right)-\varphi^{\prime}\left(\nu_{i_{k}}\right)\right]+\varepsilon / 2<\varepsilon
\end{aligned}
$$

Thus $\lim _{k} \varphi\left(\mu_{i_{k}}\right)=\varphi(\mu)$ as required.

From Lemma 5, and noting that the set $\left\{\mu^{\prime} \mid \mu \stackrel{a}{\rightarrow} \mu^{\prime}\right\}$ is compact for each $\mu$ and $a$, the supremum in the semantic definition of $\langle a\rangle \varphi$ can be replaced by maximum; that is,

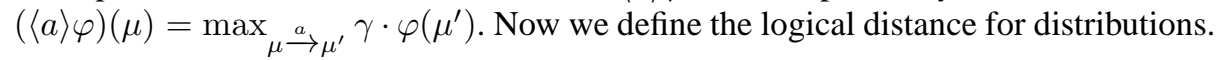

Definition 13. The logic distance of $\mu$ and $\nu$ in Dist $(S)$ of an input enabled automaton is defined by

$$
D_{l}^{\mathcal{A}}(\mu, \nu)=\sup _{\varphi \in \mathcal{L}_{m}}|\varphi(\mu)-\varphi(\nu)| .
$$

The logic distance for a general probabilistic automaton can be defined in terms of the input enabled extension; that is, $D_{l}^{\mathcal{A}}(\mu, \nu):=D_{l}^{\mathcal{A}_{\perp}}(\mu, \nu)$. We always omit the superscripts for simplicity.

Now we can show that the logic distance exactly coincides with bisimulation distance for any distributions.

Theorem 4. $D_{b}=D_{l}$.

Proof. As both $D_{b}$ and $D_{l}$ are defined in terms of the input enabled extension of automata, we only need to prove the result for input enabled case. Let $\mu, \nu \in \operatorname{Dist}(S)$. We first prove $D_{b}(\mu, \nu) \geq D_{l}(\mu, \nu)$. It suffices to show by structural induction that for any $\varphi \in \mathcal{L}_{m},|\varphi(\mu)-\varphi(\nu)| \leq D_{b}(\mu, \nu)$. There are five cases to consider.

- $\varphi \equiv \mathcal{B}$ for some $\mathcal{B} \subseteq 2^{A P}$. Then $|\varphi(\mu)-\varphi(\nu)|=\left|\sum_{A \in \mathcal{B}}[\mu(A)-\nu(A)]\right| \leq$ $d_{A P}(\mu, \nu) \leq D_{b}(\mu, \nu)$ by Theorem 2 .

- $\varphi \equiv \varphi^{\prime} \oplus p$. Assume $\varphi^{\prime}(\mu) \geq \varphi^{\prime}(\nu)$. Then $\varphi(\mu) \geq \varphi(\nu)$. By induction, we have $\varphi^{\prime}(\mu)-\varphi^{\prime}(\nu) \leq D_{b}(\mu, \nu)$. Thus

$$
|\varphi(\mu)-\varphi(\nu)|=\min \left\{\varphi^{\prime}(\mu)+p, 1\right\}-\min \left\{\varphi^{\prime}(\nu)+p, 1\right\} \leq \varphi^{\prime}(\mu)-\varphi^{\prime}(\nu) \leq D_{b}(\mu, \nu) .
$$

- $\varphi \equiv \neg \varphi^{\prime}$. By induction, we have $\left|\varphi^{\prime}(\mu)-\varphi^{\prime}(\nu)\right| \leq D_{b}(\mu, \nu)$, thus $|\varphi(\mu)-\varphi(\nu)|=$ $\left|1-\varphi^{\prime}(\mu)-1+\varphi^{\prime}(\nu)\right| \leq D_{b}(\mu, \nu)$ as well.

- $\varphi \equiv \bigwedge_{i \in I} \varphi_{i}$. Assume $\varphi(\mu) \geq \varphi(\nu)$. For any $\varepsilon>0$, let $j \in I$ such that $\varphi_{j}(\nu) \leq$ $\varphi(\nu)+\varepsilon$. By induction, we have $\left|\varphi_{j}(\mu)-\varphi_{j}(\nu)\right| \leq D_{b}(\mu, \nu)$. Then

$$
|\varphi(\mu)-\varphi(\nu)| \leq \varphi_{j}(\mu)-\varphi_{j}(\nu)+\varepsilon \leq D_{b}(\mu, \nu)+\varepsilon,
$$

and $|\varphi(\mu)-\varphi(\nu)| \leq D_{b}(\mu, \nu)$ from the arbitrariness of $\varepsilon$. 
- $\varphi \equiv\langle a\rangle \varphi^{\prime}$. Assume $\varphi(\mu) \geq \varphi(\nu)$. Let $\mu_{*}^{\prime} \in \operatorname{Dist}(S)$ such that $\mu \stackrel{a}{\rightarrow} \mu_{*}^{\prime}$ and $\gamma \cdot \varphi^{\prime}\left(\mu_{*}^{\prime}\right)=\varphi(\mu)$. From Theorem 2, we have $\mu \sim_{D_{b}(\mu, \nu)} \nu$. Thus there exists $\nu_{*}^{\prime}$ such that $\nu \stackrel{a}{\rightarrow} \nu_{*}^{\prime}$ and $\mu_{*}^{\prime} \sim_{D_{b}(\mu, \nu) / \gamma} \nu_{*}^{\prime}$. Hence $\gamma \cdot D_{b}\left(\mu_{*}^{\prime}, \nu_{*}^{\prime}\right) \leq D_{b}(\mu, \nu)$, and

$$
|\varphi(\mu)-\varphi(\nu)| \leq \gamma \cdot\left[\varphi^{\prime}\left(\mu_{*}^{\prime}\right)-\varphi^{\prime}\left(\nu_{*}^{\prime}\right)\right] \leq \gamma \cdot D_{b}\left(\mu_{*}^{\prime}, \nu_{*}^{\prime}\right) \leq D_{b}(\mu, \nu)
$$

where the second inequality is from induction.

Now we turn to the proof of $D_{b}(\mu, \nu) \leq D_{l}(\mu, \nu)$. We will achieve this by showing that the symmetric relations $R_{\varepsilon}=\left\{(\mu, \nu) \mid D_{l}(\mu, \nu) \leq \varepsilon\right\}$, where $\varepsilon \geq 0$, constitute an approximate bisimulation. Let $\mu R_{\varepsilon} \nu$ for some $\varepsilon \geq 0$. First, for any $\mathcal{B} \subseteq 2^{A P}$ we have

$$
\left|\sum_{A \in \mathcal{B}} \mu(A)-\sum_{A \in \mathcal{B}} \nu(A)\right|=|\mathcal{B}(\mu)-\mathcal{B}(\nu)| \leq D_{l}(\mu, \nu) \leq \varepsilon .
$$

Thus $d_{A P}(\mu, \nu) \leq \varepsilon$ as well. Now suppose $\mu \stackrel{a}{\rightarrow} \mu^{\prime}$ for some $\mu^{\prime}$. We have to show that there is some $\nu^{\prime}$ with $\nu \stackrel{a}{\rightarrow} \nu^{\prime}$ and $D_{l}\left(\mu^{\prime}, \nu^{\prime}\right) \leq \varepsilon / \gamma$. Consider the set

$$
\mathcal{K}=\left\{\omega \in \operatorname{Dist}(S) \mid \nu \stackrel{a}{\rightarrow} \omega \text { and } D_{l}\left(\mu^{\prime}, \omega\right)>\varepsilon / \gamma\right\} .
$$

For each $\omega \in \mathcal{K}$, there must be some $\varphi_{\omega}$ such that $\left|\varphi_{\omega}\left(\mu^{\prime}\right)-\varphi_{\omega}(\omega)\right|>\varepsilon / \gamma$. As our logic includes the operator $\neg$, we can always assume that $\varphi_{\omega}\left(\mu^{\prime}\right)>\varphi_{\omega}(\omega)+\varepsilon / \gamma$. Let $p=\sup _{\omega \in \mathcal{K}} \varphi_{\omega}\left(\mu^{\prime}\right)$. Let

$$
\varphi_{\omega}^{\prime}=\varphi_{\omega} \oplus\left[p-\varphi_{\omega}\left(\mu^{\prime}\right)\right], \quad \varphi^{\prime}=\bigwedge_{\omega \in \mathcal{K}} \varphi_{\omega}^{\prime}, \quad \text { and } \quad \varphi=\langle a\rangle \varphi^{\prime}
$$

Then from the assumption that $D_{l}(\mu, \nu) \leq \varepsilon$, we have $|\varphi(\mu)-\varphi(\nu)| \leq \varepsilon$. Furthermore, we check that for any $\omega \in \mathcal{K}$,

$$
\varphi_{\omega}^{\prime}\left(\mu^{\prime}\right)=\varphi_{\omega}\left(\mu^{\prime}\right) \oplus\left[p-\varphi_{\omega}\left(\mu^{\prime}\right)\right]=p .
$$

Thus $\varphi(\mu) \geq \gamma \cdot \varphi^{\prime}\left(\mu^{\prime}\right)=\gamma \cdot p$.

Let $\nu^{\prime}$ be the distribution such that $\nu \stackrel{a}{\rightarrow} \nu^{\prime}$ and $\varphi(\nu)=\gamma \cdot \varphi^{\prime}\left(\nu^{\prime}\right)$. We are going to show that $\nu^{\prime} \notin \mathcal{K}$, and then $D_{l}\left(\mu^{\prime}, \nu^{\prime}\right) \leq \varepsilon / \gamma$ as required. For this purpose, assume conversely that $\nu^{\prime} \in \mathcal{K}$. Then

$$
\begin{aligned}
\varphi(\nu) & =\gamma \cdot \varphi^{\prime}\left(\nu^{\prime}\right) \leq \gamma \cdot \varphi_{\nu^{\prime}}^{\prime}\left(\nu^{\prime}\right) \leq \gamma \cdot\left[\varphi_{\nu^{\prime}}\left(\nu^{\prime}\right)+p-\varphi_{\nu^{\prime}}\left(\mu^{\prime}\right)\right] \\
& <\gamma \cdot p-\varepsilon \leq \varphi(\mu)-\varepsilon
\end{aligned}
$$

contradicting the fact that $|\varphi(\mu)-\varphi(\nu)| \leq \varepsilon$.

We have proven that $\left\{R_{\varepsilon} \mid \varepsilon \geq 0\right\}$ is an approximate bisimulation. Thus $\mu \sim_{\varepsilon} \nu$, and so $D_{b}(\mu, \nu) \leq \varepsilon$, whenever $D_{l}(\mu, \nu) \leq \varepsilon$. So we have $D_{b}(\mu, \nu) \leq D_{l}(\mu, \nu)$ from the arbitrariness of $\varepsilon$. 


\subsection{A Fixed Point-Based Approach}

In the following, we denote by $\mathcal{M}$ the set of pseudometrics over $\operatorname{Dist}(S)$. Denote by $\mathbf{0}$ the zero pseudometric which assigns 0 to each pair of distributions. For any $d, d^{\prime} \in \mathcal{M}$, we write $d \leq d^{\prime}$ if $d(\mu, \nu) \leq d^{\prime}(\mu, \nu)$ for any $\mu$ and $\nu$. Obviously $\leq$ is a partial order, and $(\mathcal{M}, \leq)$ is a complete lattice.

Definition 14. Let $\mathcal{A}=(S, A c t, \rightarrow, L, \alpha)$ be an input enabled probabilistic automaton. We define the function $F: \mathcal{M} \rightarrow \mathcal{M}$ as follows. For any $\mu, \nu \in \operatorname{Dist}(S)$,

$$
\begin{aligned}
F(d)(\mu, \nu)=\max _{a \in A c t}\{ & d_{A P}(\mu, \nu), \\
& \sup _{\mu \stackrel{a}{\rightarrow} \mu^{\prime} \nu \stackrel{a}{\rightarrow} \nu^{\prime}} \gamma \cdot d\left(\mu^{\prime}, \nu^{\prime}\right), \sup _{\nu \stackrel{a}{\rightarrow} \nu^{\prime}} \inf _{\mu} \stackrel{a}{\rightarrow} \mu^{\prime} \\
& \left.\gamma \cdot d\left(\mu^{\prime}, \nu^{\prime}\right)\right\} .
\end{aligned}
$$

Then, $F$ is monotonic with respect to $\leq$, and by Knaster-Tarski theorem, $F$ has a least fixed point, denoted $D_{f}^{\mathcal{A}}$, given by

$$
D_{f}^{\mathcal{A}}=\bigvee_{n=0}^{\infty} F^{n}(\mathbf{0})
$$

Once again, the fixed point-based distance for a general probabilistic automaton can be defined in terms of the input enabled extension; that is, $D_{f}^{\mathcal{A}}(\mu, \nu):=D_{f}^{\mathcal{A}_{\perp}}(\mu, \nu)$. We always omit the superscripts for simplicity.

Similar to Lemma 5, we can show that the supremum (resp. infimum) in Definiton 14 can be replaced by maximum (resp. minimum). Now we show that $D_{f}$ coincides with $D_{b}$.

Theorem 5. $D_{f}=D_{b}$.

As both $D_{f}$ and $D_{b}$ are defined in terms of the input enabled extension of automata, we only need to prove Theorem 5 for input enabled case, which will be obtained by combining Lemma 6 and Lemma 7 below.

Lemma 6. For input enabled probabilistic automata, $D_{f} \leq D_{b}$.

Proof. It suffices to prove by induction that for any $n \geq 0, F^{n}(\mathbf{0}) \leq D_{b}$. The case of $n=0$ is trivial. Suppose $F^{n}(\mathbf{0}) \leq D_{b}$ for some $n \geq 0$. Then for any $a \in A c t$ and any $\mu, \nu$, we have

(1) $d_{A P}(\mu, \nu) \leq D_{b}(\mu, \nu)$ by the fact that $\mu \sim_{D_{b}(\mu, \nu)} \nu$;

(2) Note that $\mu \sim_{D_{b}(\mu, \nu)} \nu$. Whenever $\mu \stackrel{a}{\rightarrow} \mu^{\prime}$, we have $\nu \stackrel{a}{\rightarrow} \nu^{\prime}$ for some $\nu^{\prime}$ such that $\mu^{\prime} \sim_{D_{b}(\mu, \nu) / \gamma} \nu^{\prime}$, and hence $\gamma \cdot D_{b}\left(\mu^{\prime}, \nu^{\prime}\right) \leq D_{b}(\mu, \nu)$. That, together with the assumption $F^{n}(\mathbf{0}) \leq D_{b}$, implies

$$
\max _{\mu \stackrel{a}{\rightarrow} \mu^{\prime} \nu \stackrel{a}{\rightarrow} \nu^{\prime}} \gamma \cdot F^{n}(\mathbf{0})\left(\mu^{\prime}, \nu^{\prime}\right) \leq D_{b}(\mu, \nu) .
$$

The symmetric form can be similarly proved.

Summing up (1) and (2), we have $F^{n+1}(\mathbf{0}) \leq D_{b}$.

The opposite direction is summarized in the following lemma. The proof is technically involved so we put it into the appendix.

Lemma 7. For input enabled probabilistic automata, $D_{b} \leq D_{f}$. 


\subsection{Comparison with State-Based Metrics}

In this section, we prove that our distribution-based bisimulation metric is lower bounded by the state-based game bisimulation metrics [8] for MDPs. This game bisimulation metric is particularly attractive as it preserves probabilistic reachability, long-run, and discounted average behaviors [5]. We first recall the definition of state-based game bisimulation metrics [8] for MDPs:

Definition 15. Given $\mu, \nu \in \operatorname{Dist}(S), \mu \otimes \nu$ is defined as the set of weight functions $\lambda: S \times S \rightarrow[0,1]$ such that for any $s, t \in S$,

$$
\sum_{s \in S} \lambda(s, t)=\nu(t) \quad \text { and } \quad \sum_{t \in S} \lambda(s, t)=\mu(s) .
$$

Given a metric d defined on $S$, we lift it to Dist $(S)$ by defining

$$
d(\mu, \nu)=\inf _{\lambda \in \mu \otimes \nu}\left(\sum_{s, t \in S} \lambda(s, t) \cdot d(s, t)\right) .
$$

Actually the infimum in the above definition is attainable.

Definition 16. We define the function $f: \mathcal{M} \rightarrow \mathcal{M}$ as follows. For any $s, t \in S$,

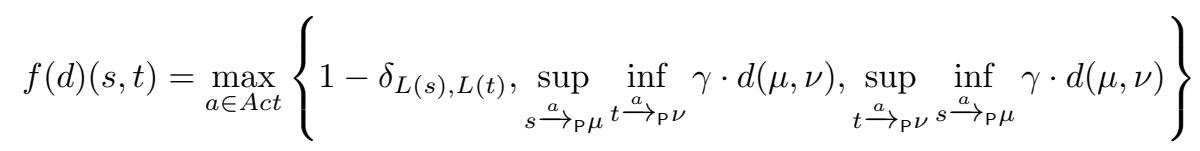

where $\delta_{L(s), L(t)}=1$ if $L(s)=L(t)$, and 0 otherwise. We take inf $\emptyset=1$ and $\sup \emptyset=0$. Again, $f$ is monotonic with respect to $\leq$, and by Knaster-Tarski theorem, $F$ has a least fixed point, denoted $d_{f}$, given by

$$
d_{f}=\bigvee_{n=0}^{\infty} f^{n}(\mathbf{0}) .
$$

Now we can prove the quantitative extension of Lemma 1. Without loss of any generality, we assume that $\mathcal{A}$ itself is input enabled. Let $d_{n}=f^{n}(\mathbf{0})$ and $D_{n}=F^{n}(\mathbf{0})$ in Definition 14.

Lemma 8. For any $n \geq 1, d_{A P}(\mu, \nu) \leq d_{n}(\mu, \nu)$.

Proof. Let $\lambda$ be the weight function such that $d_{n}(\mu, \nu)=\sum_{s, t \in S} \lambda(s, t) \cdot d_{n}(s, t)$. Since $d_{n}(s, t) \geq 1-\delta_{L(s), L(t)}$, we have

$$
d_{n}(\mu, \nu) \geq 1-\sum_{s, t: L(s)=L(t)} \lambda(s, t) .
$$

On the other hand, for any $A \subseteq A P$, recall that $S(A)=\{s \in S \mid L(s)=A\}$. Then

$$
\begin{aligned}
\mu(A)-\nu(A) & =\sum_{s \in S(A)} \mu(s)-\sum_{t \in S(A)} \nu(t) \\
& =\sum_{s \in S(A)} \sum_{t \notin S(A)} \lambda(s, t)-\sum_{t \in S(A)} \sum_{s \notin S(A)} \lambda(s, t) .
\end{aligned}
$$


Let $\mathcal{B} \subseteq 2^{A P}$ such that $d_{A P}(\mu, \nu)=\sum_{A \in \mathcal{B}}[\mu(A)-\nu(A)]$. Then

$$
d_{A P}(\mu, \nu) \leq \sum_{A \in \mathcal{B}} \sum_{s \in S(A)} \sum_{t \notin S(A)} \lambda(s, t) \leq \sum_{s, t: L(s) \neq L(t)} \lambda(s, t),
$$

and the result follows.

Theorem 6. Let $\mathcal{A}$ be a probabilistic automaton. Then $D_{f} \leq d_{f}$.

Proof. We prove by induction on $n$ that $D_{n}(\mu, \nu) \leq d_{n}(\mu, \nu)$ for any $\mu, \nu \in \operatorname{Dist}(S)$ and $n \geq 0$. The case $n=0$ is obvious. Suppose the result holds for some $n-1 \geq 0$. Then from Lemma 8, we need only to show that for any $\mu \stackrel{a}{\rightarrow} \mu^{\prime}$ there exists $\nu \stackrel{a}{\rightarrow} \nu^{\prime}$ such that $\gamma \cdot D_{n-1}\left(\mu^{\prime}, \nu^{\prime}\right) \leq d_{n}(\mu, \nu)$.

Let $\mu \stackrel{a}{\rightarrow} \mu^{\prime}$. Then for any $s \in S, s \stackrel{a}{\rightarrow} \mathrm{P} \mu_{s}$ with $\mu^{\prime}=\sum_{s \in S} \mu(s) \cdot \mu_{s}$. By definition of $d_{n}$, for any $t \in S$, we have $t \stackrel{a}{\rightarrow} \mathrm{p} \nu_{t}$ such that $\gamma \cdot d_{n-1}\left(\mu_{s}, \nu_{t}\right) \leq d_{n}(s, t)$. Thus $\nu \stackrel{a}{\rightarrow} \nu^{\prime}:=\sum_{t \in S} \nu(t) \cdot \nu_{t}$, and by induction, $D_{n-1}\left(\mu^{\prime}, \nu^{\prime}\right) \leq d_{n-1}\left(\mu^{\prime}, \nu^{\prime}\right)$. Now it suffices to prove $\gamma \cdot d_{n-1}\left(\mu^{\prime}, \nu^{\prime}\right) \leq d_{n}(\mu, \nu)$.

Let $\lambda \in \mu \otimes \nu$ and $\gamma_{s, t} \in \mu_{s} \otimes \nu_{t}$ be the weight functions such that

$$
d_{n}(\mu, \nu)=\sum_{s, t \in S} \lambda(s, t) \cdot d_{n}(s, t), d_{n-1}\left(\mu_{s}, \nu_{t}\right)=\sum_{u, v \in S} \gamma_{s, t}(u, v) \cdot d_{n-1}(u, v) .
$$

Then

$$
\begin{aligned}
d_{n}(\mu, \nu) & \geq \gamma \cdot \sum_{s, t \in S} \lambda(s, t) \cdot d_{n-1}\left(\mu_{s}, \nu_{t}\right) \\
& =\gamma \cdot \sum_{u, v \in S} \sum_{s, t \in S} \lambda(s, t) \gamma_{s, t}(u, v) \cdot d_{n-1}(u, v) .
\end{aligned}
$$

We need to show that the function $\eta(u, v):=\sum_{s, t \in S} \lambda(s, t) \gamma_{s, t}(u, v)$ is a weight function for $\mu^{\prime}$ and $\nu^{\prime}$. It is easy to check that

$$
\begin{aligned}
\sum_{u} \eta(u, v) & =\sum_{s, t \in S} \lambda(s, t) \sum_{u} \gamma_{s, t}(u, v)=\sum_{s, t \in S} \lambda(s, t) \nu_{t}(v) \\
& =\sum_{t \in S} \nu(t) \nu_{t}(v)=\nu^{\prime}(v) .
\end{aligned}
$$

Similarly, we have $\sum_{v} \eta(u, v)=\mu^{\prime}(u)$.

Example 2. Consider Fig. 1, and assume $\varepsilon_{1} \geq \varepsilon_{2}>0$. Applying the definition of $D_{b}$, it is easy to check that $D_{b}\left(\delta_{q}, \delta_{q^{\prime}}\right)=0.5\left(\varepsilon_{1}-\varepsilon_{2}\right) \gamma$. By our results, we have $D_{l}\left(\delta_{q}, \delta_{q^{\prime}}\right)=D_{f}\left(\delta_{q}, \delta_{q^{\prime}}\right)=D_{b}\left(\delta_{q}, \delta_{q^{\prime}}\right)$. Note that for the discounting case $\gamma<1$, difference far in the future will have less influence in the distance.

We further compute the distance under state-based bisimulation metrics (see [16] for example). Assume that $\gamma=1$. One first compute the distance between $r_{1}$ and $r^{\prime}$ being $\frac{1}{6}+\varepsilon_{1}$, between $r_{2}$ and $r^{\prime}$ being $\frac{1}{6}+\varepsilon_{2}$. Then, the state-based bisimulation metric between $q$ and $q^{\prime}$ is $\frac{1}{6}+0.5\left(\varepsilon_{1}+\varepsilon_{2}\right)$, which can be obtained by lifting the state-based metrics. 


\subsection{Comparison with Equivalence Metric}

Note that we can easily extend the equivalence relation defined in Definition 5 to a notion of equivalence metric:

Definition 17 (Equivalence Metric). Let $\mathcal{A}_{i}=\left(S_{i}\right.$, Act $\left._{i}, \rightarrow_{i}, L_{i}, \alpha_{i}\right)$ with $i=1,2$ be two reactive automata with $A c t_{1}=A c t_{2}=: A c t$, and $F_{i}=\left\{s \in S_{i} \mid L(s)=A P\right\}$ the set of final states for $\mathcal{A}_{i}$. We say $\mathcal{A}_{1}$ and $\mathcal{A}_{2}$ are $\varepsilon$-equivalent, denoted $\mathcal{A}_{1} \sim_{\varepsilon}^{d} \mathcal{A}_{2}$, iffor any input word $w=a_{1} a_{2} \ldots a_{n},\left|\mathcal{A}_{1}(w)-\mathcal{A}_{2}(w)\right| \leq \varepsilon$. Furthermore, the equivalence distance between $\mathcal{A}_{1}$ and $\mathcal{A}_{2}$ is defined by $D_{d}\left(\mathcal{A}_{1}, \mathcal{A}_{2}\right):=\inf \left\{\varepsilon \geq 0 \mid \mathcal{A}_{1} \sim_{\varepsilon}^{d} \mathcal{A}_{2}\right\}$.

Now we show that for reactive automata, the equivalence metric $D_{d}$ coincide with our undiscounted bisimulation metric $D_{b}$, which may be regarded as a quantitative extension of Lemma 2.

Proposition 1. Let $\mathcal{A}_{1}$ and $\mathcal{A}_{2}$ be two reactive automata with the same set of actions Act. Let the discount factor $\gamma=1$. Then $D_{d}\left(\mathcal{A}_{1}, \mathcal{A}_{2}\right)=D_{b}\left(\alpha_{1}, \alpha_{2}\right)$ where $D_{b}$ is defined in the direct sum of $\mathcal{A}_{1}$ and $\mathcal{A}_{2}$.

Proof. We first show that $D_{d}\left(\mathcal{A}_{1}, \mathcal{A}_{2}\right) \leq D_{b}\left(\alpha_{1}, \alpha_{2}\right)$. For each input word $w=$ $a_{1} a_{2} \ldots a_{n}$, it is easy to check that $\mathcal{A}_{i}(w)=\varphi\left(\alpha_{i}\right)$ where $\varphi=\left\langle a_{1}\right\rangle\left\langle a_{2}\right\rangle \ldots\left\langle a_{n}\right\rangle\left(F_{1} \cup\right.$ $\left.F_{2}\right)$. As we have shown that $D_{b}=D_{l}$, it holds $\left|\mathcal{A}_{1}(w)-\mathcal{A}_{2}(w)\right| \leq D_{b}\left(\alpha_{1}, \alpha_{2}\right)$, and hence $\mathcal{A}_{1} \sim_{D_{b}\left(\alpha_{1}, \alpha_{2}\right)}^{d} \mathcal{A}_{2}$. Then $D_{d}\left(\mathcal{A}_{1}, \mathcal{A}_{2}\right) \leq D_{b}\left(\alpha_{1}, \alpha_{2}\right)$ by definition.

Now we turn to the proof of $D_{d}\left(\mathcal{A}_{1}, \mathcal{A}_{2}\right) \geq D_{b}\left(\alpha_{1}, \alpha_{2}\right)$. First we show that

$$
R_{\varepsilon}=\left\{(\mu, \nu) \mid \mu \in \operatorname{Dist}\left(S_{1}\right), \nu \in \operatorname{Dist}\left(S_{2}\right), \mathcal{A}_{1}^{\mu} \sim_{\varepsilon}^{d} \mathcal{A}_{2}^{\nu}\right\}
$$

is an approximate bisimulation. Here for a probabilistic automaton $\mathcal{A}$, we denote by $\mathcal{A}^{\mu}$ the automaton which is the same as $\mathcal{A}$ except that the initial distribution is replaced by $\mu$. Let $\mu R_{\varepsilon} \nu$. Since $L(s) \in\{\emptyset, A P\}$ for all $s \in S_{1} \cup S_{2}$, we have $\mu(A P)+\mu(\emptyset)=$ $\nu(A P)+\nu(\emptyset)=1$. Thus

$$
d_{A P}(\mu, \nu)=|\mu(A P)-\nu(A P)|=\left|\mu\left(F_{1}\right)-\nu\left(F_{2}\right)\right| .
$$

Note that $\mu\left(F_{1}\right)=\mathcal{A}_{1}^{\mu}(e)$ and $\nu\left(F_{2}\right)=\mathcal{A}_{2}^{\nu}(e)$, where $e$ is the empty string. Then $d_{A P}(\mu, \nu)=\left|\mathcal{A}_{1}^{\mu}(e)-\mathcal{A}_{2}^{\nu}(e)\right| \leq \varepsilon$.

Let $\mu \stackrel{a}{\rightarrow} \mu^{\prime}$ and $\nu \stackrel{a}{\rightarrow} \nu^{\prime}$. We need to show $\mu^{\prime} R_{\varepsilon} \nu^{\prime}$, that is, $\mathcal{A}_{1}^{\mu^{\prime}} \sim_{\varepsilon}^{d} \mathcal{A}_{2}^{\nu^{\prime}}$. For any $w \in A c t^{*}$, note that $\mathcal{A}_{1}^{\mu^{\prime}}(w)=\mathcal{A}_{1}^{\mu}(a w)$. Then

$$
\left|\mathcal{A}_{1}^{\mu^{\prime}}(w)-\mathcal{A}_{2}^{\nu^{\prime}}(w)\right|=\left|\mathcal{A}_{1}^{\mu}(a w)-\mathcal{A}_{2}^{\nu}(a w)\right| \leq \varepsilon,
$$

and hence $\mathcal{A}_{1}^{\mu^{\prime}} \sim_{\varepsilon}^{d} \mathcal{A}_{2}^{\nu^{\prime}}$ as required.

Having proven that $R_{\varepsilon}$ is an approximate bisimulation, we know $\mathcal{A}_{1} \sim_{\varepsilon}^{d} \mathcal{A}_{2}$ implies $\alpha_{1} \sim_{\varepsilon} \alpha_{2}$. Thus $D_{d}\left(\mathcal{A}_{1}, \mathcal{A}_{2}\right)=\inf \left\{\varepsilon \mid \mathcal{A}_{1} \sim_{\varepsilon}^{d} \mathcal{A}_{2}\right\} \geq \inf \left\{\alpha_{1} \sim_{\varepsilon} \alpha_{2}\right\}=D_{b}\left(\alpha_{1}, \alpha_{2}\right)$. 


\section{Discussion and Future Work}

In this paper, we considered Segala's automata, and proposed a novel notion of bisimulation by joining the existing notions of equivalence and bisimilarities. We have demonstrated the utility of our definition by studying distribution-based bisimulation metrics, which have been extensively studied for MDPs.

As future work we would like to identify further solutions and techniques developed in one area that could inspire solutions for the corresponding problems in the other area. This includes for instance decision algorithm developed for equivalence checking [30,24], extensions to simulations, and compositional verification for probabilistic automata.

\section{References}

1. G. Bacci, G. Bacci, K. G. Larsen, and R. Mardare. On-the-Fly Exact Computation of Bisimilarity Distances. In N. Piterman and S. A. Smolka, editors, TACAS, volume 7795 of Lecture Notes in Computer Science, pages 1-15. Springer, 2013.

2. C. Baier, B. Engelen, and M. E. Majster-Cederbaum. Deciding Bisimilarity and Similarity for Probabilistic Processes. J. Comput. Syst. Sci., 60(1):187-231, 2000.

3. R. Bellman. Dynamic Programming. Princeton University Press, 1957.

4. S. Cattani and R. Segala. Decision Algorithms for Probabilistic Bisimulation. In L. Brim, P. Jancar, M. Kretínský, and A. Kucera, editors, CONCUR, volume 2421 of Lecture Notes in Computer Science, pages 371-385. Springer, 2002.

5. K. Chatterjee, L. de Alfaro, R. Majumdar, and V. Raman. Algorithms for game metrics (full version). Logical Methods in Computer Science, 6(3), 2010.

6. D. Chen, F. van Breugel, and J. Worrell. On the Complexity of Computing Probabilistic Bisimilarity. In L. Birkedal, editor, FoSSaCS, volume 7213 of Lecture Notes in Computer Science, pages 437-451. Springer, 2012.

7. G. Comanici, P. Panangaden, and D. Precup. On-the-Fly Algorithms for Bisimulation Metrics. In QEST, pages 94-103. IEEE Computer Society, 2012.

8. L. de Alfaro, R. Majumdar, V. Raman, and M. Stoelinga. Game relations and metrics. In LICS, pages 99-108. IEEE Computer Society, 2007.

9. Y. Deng, R. J. van Glabbeek, M. Hennessy, and C. Morgan. Testing finitary probabilistic processes. In M. Bravetti and G. Zavattaro, editors, CONCUR, volume 5710 of Lecture Notes in Computer Science, pages 274-288. Springer, 2009.

10. J. Desharnais, V. Gupta, R. Jagadeesan, and P. Panangaden. Metrics for Labeled Markov Systems. In J. C. M. Baeten and S. Mauw, editors, CONCUR, volume 1664 of Lecture Notes in Computer Science, pages 258-273. Springer, 1999.

11. J. Desharnais, V. Gupta, R. Jagadeesan, and P. Panangaden. Metrics for labelled markov processes. Theor. Comput. Sci., 318(3):323-354, 2004.

12. J. Desharnais, V. Gupta, R. Jagadeesan, and P. Panangaden. Weak bisimulation is sound and complete for pCTL ${ }^{*}$. Inf. Comput., 208(2):203-219, 2010.

13. J. Desharnais, F. Laviolette, and M. Tracol. Approximate Analysis of Probabilistic Processes: Logic, Simulation and Games. In QEST, pages 264-273. IEEE Computer Society, 2008.

14. L. Doyen, T. A. Henzinger, and J.-F. Raskin. Equivalence of Labeled Markov Chains. Int. J. Found. Comput. Sci., 19(3):549-563, 2008.

15. C. Eisentraut, H. Hermanns, and L. Zhang. On Probabilistic Automata in Continuous Time. In LICS, pages 342-351. IEEE Computer Society, 2010.

16. N. Ferns, P. Panangaden, and D. Precup. Bisimulation Metrics for Continuous Markov Decision Processes. SIAM J. Comput., 40(6):1662-1714, 2011. 
17. H. Fu. Computing Game Metrics on Markov Decision Processes. In A. Czumaj, K. Mehlhorn, A. M. Pitts, and R. Wattenhofer, editors, ICALP (2), volume 7392 of Lecture Notes in Computer Science, pages 227-238. Springer, 2012.

18. A. Giacalone, C. Jou, and S. Smolka. Algebraic reasoning for probabilistic concurrent systems. In IFIP TC2 Working Conference on Programming Concepts and Methods, page 443458. North-Holland, 1990.

19. E. M. Hahn, Y. Li, S. Schewe, A. Turrini, and Z. Lijun. IsCASMC: A web-based probabilistic model checker. In $F M, 2014$. To appear.

20. M. Hennessy. Exploring probabilistic bisimulations, part I. Formal Asp. Comput., 24(46):749-768, 2012.

21. H. Hermanns, A. Parma, R. Segala, B. Wachter, and L. Zhang. Probabilistic Logical Characterization. Inf. Comput., 209(2):154-172, 2011.

22. H. Hermanns and A. Turrini. Deciding Probabilistic Automata Weak Bisimulation in Polynomial Time. In D. D'Souza, T. Kavitha, and J. Radhakrishnan, editors, FSTTCS, volume 18 of LIPIcs, pages 435-447. Schloss Dagstuhl - Leibniz-Zentrum fuer Informatik, 2012.

23. J.-P. Katoen, I. S. Zapreev, E. M. Hahn, H. Hermanns, and D. N. Jansen. The ins and outs of the probabilistic model checker mrmc. Perform. Eval., 68(2):90-104, 2011.

24. S. Kiefer, A. S. Murawski, J. Ouaknine, B. Wachter, and J. Worrell. Language Equivalence for Probabilistic Automata. In G. Gopalakrishnan and S. Qadeer, editors, CAV, volume 6806 of Lecture Notes in Computer Science, pages 526-540. Springer, 2011.

25. S. Kiefer, A. S. Murawski, J. Ouaknine, B. Wachter, and J. Worrell. On the Complexity of the Equivalence Problem for Probabilistic Automata. In L. Birkedal, editor, FoSSaCS, volume 7213 of Lecture Notes in Computer Science, pages 467-481. Springer, 2012.

26. M. Z. Kwiatkowska, G. Norman, and D. Parker. PRISM 4.0: Verification of Probabilistic Real-Time Systems. In G. Gopalakrishnan and S. Qadeer, editors, $C A V$, volume 6806 of Lecture Notes in Computer Science, pages 585-591. Springer, 2011.

27. A. Parma and R. Segala. Logical Characterizations of Bisimulations for Discrete Probabilistic Systems. In H. Seidl, editor, FoSSaCS, volume 4423 of Lecture Notes in Computer Science, pages 287-301. Springer, 2007.

28. M. Rabin. Probabilistic automata. Information and Control, 6(3):230245, 1963.

29. R. Segala. Modeling and Verification of Randomized Distributed Realtime Systems. PhD thesis, MIT, 1995.

30. W. Tzeng. A polynomial-time algorithm for the equivalence of probabilistic automata. SIAM Journal on Computing, 21(2):216227, 1992.

31. F. van Breugel, B. Sharma, and J. Worrell. Approximating a Behavioural Pseudometric Without Discount for Probabilistic Systems. In H. Seidl, editor, FoSSaCS, volume 4423 of Lecture Notes in Computer Science, pages 123-137. Springer, 2007.

32. M. Ying. Topology in Process Calculus: Approximate Correctness and Infinite Evolution of Concurrent Programs. Springer-Verlag, New York, 2001.

33. M. Ying. Bisimulation indexes and their applications. Theoretical Computer Science, 275:168, 2002.

34. M. Ying and M. Wirsing. Approximate bisimilarity. In Algebraic Methodology and Software Technology, pages 309-322. Springer-Verlag, Berlin, 2000.

\section{A Omitted Proofs}

\section{A.1 Proof of Lemma 7}

To prove Lemma 7, we first introduce the notion of bounded approximation bisimulations. 
Definition 18. Let $\mathcal{A}$ be an input enabled probabilistic automaton. We define symmetric relations

- $\stackrel{\varepsilon}{\sim}_{0}:=\operatorname{Dist}(S) \times \operatorname{Dist}(S)$ for any $\varepsilon \geq 0$

- for $n \geq 0, \mu \stackrel{\varepsilon}{\sim} n+1$ if $1 \leq \varepsilon$, and whenever $\mu \stackrel{a}{\rightarrow} \mu^{\prime}$, there exists $\nu \stackrel{a}{\rightarrow} \nu^{\prime}$ for some $\nu^{\prime}$ such that $\mu^{\prime} \stackrel{\varepsilon / \gamma}{\sim}{ }_{n} \nu^{\prime}$.

- $\stackrel{\varepsilon}{\sim}:=\bigcap_{n \geq 0} \stackrel{\varepsilon}{\sim}_{n}$.

The following lemma collects some useful properties of $\underset{\sim}{\sim}$ and $\stackrel{\varepsilon}{\sim}$.

Lemma 9. 1. $\stackrel{\varepsilon}{\sim}_{n} \subseteq \stackrel{\varepsilon}{\sim}_{m}$ provided that $n \geq m$;

2. for any $n \geq 0, \stackrel{\varepsilon}{\sim}_{n} \subseteq{\stackrel{\varepsilon^{\prime}}{\sim}}_{n}$ provided that $\varepsilon \leq \varepsilon^{\prime}$;

3. for any $n \geq 0, \stackrel{\varepsilon}{\sim}_{n}$ is continuous;

4. $\stackrel{\varepsilon}{\sim}=\sim_{\varepsilon}$.

Proof. Items 1, 2, and 3 are easy by induction, and so is the $\sim_{\varepsilon} \subseteq \stackrel{\varepsilon}{\sim}$ part of Item 4 . To prove $\stackrel{\varepsilon}{\sim} \subseteq \sim_{\varepsilon}$, we show that $\{\stackrel{\varepsilon}{\sim} \mid \varepsilon \geq 0\}$ is an approximate bisimulation. Suppose $\mu \stackrel{\varepsilon}{\sim} \nu$. Then $d_{A P}(\mu, \nu) \leq \varepsilon$ by definition. Now let $\mu \stackrel{a}{\rightarrow} \mu^{\prime}$. For each $n \geq 0$, from the assumption that $\mu \stackrel{\varepsilon}{\sim}{ }_{n+1} \nu$ we have $\nu \stackrel{a}{\rightarrow} \nu_{n}$ such that $\mu^{\prime} \stackrel{\varepsilon / \gamma}{\sim}{ }_{n} \nu_{n}$. Let $\left\{\nu_{i_{k}}\right\}_{k}$ be a convergent subsequence of $\left\{\nu_{n}\right\}_{n}$ such that $\lim _{k} \nu_{i_{k}}=\nu^{\prime}$ for some $\nu^{\prime}$. Then from the continuity of $\stackrel{a}{\rightarrow}$ we have $\nu \stackrel{a}{\rightarrow} \nu^{\prime}$. We claim further that $\mu^{\prime} \stackrel{\varepsilon / \gamma}{\sim} \nu^{\prime}$. Otherwise there exists $N$ such that $\mu^{\prime} \stackrel{\varepsilon / \gamma}{\gamma_{N}} \nu^{\prime}$. Now by the continuity of $\stackrel{\varepsilon / \gamma}{\sim}{ }_{N}$, we have $\mu^{\prime}{ }^{\varepsilon / \gamma}{ }_{N} \nu_{j}$ for some $j \geq N$. This contradicts the fact that $\mu^{\prime} \stackrel{\varepsilon / \gamma}{\sim}{ }_{j} \nu_{j}$ and item 1 .

Lemma 10. For any $n \geq 0$, we have $\mu \stackrel{F^{n}(\mathbf{0})(\mu, \nu)}{\sim}{ }_{n} \nu$.

Proof. We prove this lemma by induction on $n$. The case of $n=0$ is trivial. Suppose $\mu \stackrel{F^{n}(\mathbf{0})(\mu, \nu)}{\sim}{ }_{n} \nu$ for some $n \geq 0$. Let $a \in$ Act. By definition, we have

$$
F^{n+1}(\mathbf{0})(\mu, \nu) \geq \max _{\mu \stackrel{a}{\rightarrow} \mu^{\prime} \nu \stackrel{a}{\rightarrow} \nu^{\prime}} \gamma \cdot F^{n}(\mathbf{0})\left(\mu^{\prime}, \nu^{\prime}\right)
$$

Thus for any $\mu \stackrel{a}{\rightarrow} \mu^{\prime}$, there exists $\nu \stackrel{a}{\rightarrow} \nu^{\prime}$ such that $\gamma \cdot F^{n}(\mathbf{0})\left(\mu^{\prime}, \nu^{\prime}\right) \leq F^{n+1}(\mathbf{0})(\mu, \nu)$. By induction, we know $\mu^{\prime} \stackrel{F^{n}(\mathbf{0})\left(\mu^{\prime}, \nu^{\prime}\right)}{\sim}{ }_{n} \nu^{\prime}$, thus $\mu^{f^{F^{n+1}}(\mathbf{0})(\mu, \nu) / \gamma}{ }_{n} \nu^{\prime}$ from Lemma 9(2). On the other hand, we have $F^{n+1}(\mathbf{0})(\mu, \nu) \geq d_{A P}(\mu, \nu)$ by definition. Thus we have $\mu \stackrel{F^{n+1}(\mathbf{0})(\mu, \nu)}{\sim}{ }_{n+1} \nu$.

With the two lemmas above, Lemma 7 follows easily.

Proof of Lemma 7. For any $\mu$ and $\nu$, by Lemmas 10 and 9(2), we have $\mu \stackrel{D_{f}(\mu, \nu)}{\sim}{ }_{n} \nu$ for all $n \geq 0$, so $\mu \stackrel{D_{f}(\mu, \nu)}{\sim} \nu$ by definition. Then from Lemma 9(4) we have $\mu \sim_{D_{f}(\mu, \nu)} \nu$, hence $D_{b}(\mu, \nu) \leq D_{f}(\mu, \nu)$. 